\title{
TIPIC Syndrome: Beyond the Myth of Carotidynia, a New Distinct Unclassified Entity
}

\author{
(D) Aecler, (D) M. Obadia, (D). Savatovsky, DH. Picard, (D). Charbonneau, (D). Menjot de Champfleur, (D). Naggara, DB. Carsin, \\ (D) M. Amor-Sahli, (D).P. Cottier, (D). Bensoussan, (DE. Auffray-Calvier, (D)A. Varoquaux, DS. De Gaalon, (D) C. Calazel, (D) N. Nasr, \\ (D) G. Volle, DD.C. Jianu, (D) O. Gout, DF. Bonneville, and DJ.C. Sadik
}

\begin{abstract}
BACKGROUND AND PURPOSE: The differential diagnosis of acute cervical pain includes nonvascular and vascular causes such as carotid dissection, carotid occlusion, or vasculitis. However, some patients present with unclassified vascular and perivascular changes on imaging previously reported as carotidynia. The aim of our study was to improve the description of this as yet unclassified clinico-radiologic entity.
\end{abstract}

MATERIALS AND METHODS: From January 2009 through April 2016, 47 patients from 10 centers presenting with acute neck pain or tenderness and at least 1 cervical image showing unclassified carotid abnormalities were included. We conducted a systematic, retrospective study of their medical charts and diagnostic and follow-up imaging. Two neuroradiologists independently analyzed the blinded image datasets.

RESULTS: The median patient age was 48 years. All patients presented with acute neck pain, and 8 presented with transient neurologic symptoms. Imaging showed an eccentric pericarotidian infiltration in all patients. An intimal soft plaque was noted in 16 patients, and a mild luminal narrowing was noted in 16 patients. Interreader reproducibility was excellent. All patients had complete pain resolution within a median of 13 days. At 3-month follow-up, imaging showed complete disappearance of vascular abnormalities in 8 patients, and a marked decrease in all others.

CONCLUSIONS: Our study improved the description of an unclassified, clinico-radiologic entity, which could be described by the proposed acronym: Translent Perivascular Inflammation of the Carotid artery (TIPIC) syndrome.

ABBREVIATIONS: PVI = perivascular infiltration; TIPIC = Translent Perivascular Inflammation of the Carotid artery; US = ultrasonography

C arotidynia was a clinical entity described by Fay in 1927, characterized by tenderness and pain at the level of the carotid bifurcation. Initially classified as an idiopathic neck pain

Received November 23, 2016; accepted after revision March 5, 2017.

From the Departments of Radiology (A.L., J.S., F.C., J.C.S.), and Neurology (M.O. G.V., O.G.), and Clinical Research Unit (H.P.), Fondation Ophtalmologique Adolphe de Rothschild, Paris, France; Department of Neuroradiology (N.M.d.C.), Montpellie University Hospital Center, Gui de Chauliac Hospital, Montpellier, France; Department of Radiology (B.C.), Centre Hospitalier Régional Universitaire de Rennes, Rennes, France; Department of Neuroradiology (O.N.), Centre Hospitalier SainteAnne, Paris, France; Department of Neuroradiology (M.A.-S.), Pitié-Salpêtrière Hospital, Paris, France; Centre D'imagerie Médicale Tourville (M.A.-S.), Paris, France; Department of Radiology (J.P.C.), Centre Hospitalier Régional Universitaire de Tours, Tours, France; Brain and Imaging Laboratory Unite Mixte de Recherche U930 (J.P.C.), Institut National de la Santé et de la Recherche Médicale, François-Rabelais University, Tours, France; Diagnostic and Interventional Neuroradiology Department (E.A.-C.) and Neurology Department (S.D.G.), Hôpital René et GuillaumeLaënnec, Centre Hospitalier Universitaire de Nantes, Saint-Herblain, France; Department of Radiology (J.B.), Hotel-Dieu Hospital, Paris, France; Department of Radiology (A.V.), Conception Hospital, Aix-Marseille University, Marseille, France; Departments of Neuroradiology (C.C., F.B.) and Neurology (N.N.), Hôpital PierrePaul-Riquet, Centre Hospitalier Universitaire Purpan, Toulouse, France; and Department of Neurology (D.C.J.), Victor Babes University of Medicine and Pharmacy, Timisoara, Romania.

Paper previously presented at: European Congress of Radiology, March 2-6, 2016; Vienna, Austria. syndrome in the first International Classification of Headache Disorders in $1988,{ }^{2}$ it was subsequently removed as a distinct entity in $2004 .^{3}$ Indeed, the 2 clinical signs of carotidynia were neither specific nor constant, and other causes of neck pain might have the same clinical presentation. Biousse and Bousser ${ }^{4}$ described these controversial aspects in 1994 and considered carotidynia a myth.

However, since 2000, a few case reports have described imaging abnormalities in patients presenting with tenderness and pain at the level of the carotid bifurcation. Imaging techniques like ultrasonography (US), ${ }^{5,6} \mathrm{MR}$ imaging, ${ }^{7} \mathrm{CT}$ angiography, $\left[{ }^{18} \mathrm{~F}\right]$ fluorodeoxyglucose positron-emission tomography, ${ }^{8,9}$ or associated modalities ${ }^{10}$ showed abnormal soft tissue surrounding the carotid artery and a thickened carotid wall, especially near its bifurcation. Differential diagnoses of carotid pain such as carotid

Please address correspondence to Augustin Lecler, MD, Department of Radiology, Fondation Ophtalmologique Adolphe de Rothschild, 25 Rue Manin, 75019 Paris, France; e-mail: alecler@for.paris

三 Indicates article with supplemental on-line tables.

http://dx.doi.org/10.3174/ajnr.A5214 
dissection, thyroiditis, vasculitis, head and neck inflammation or mass, sialadenitis, or cervical arthrosis were excluded. Hence, the authors suggested that a distinct entity of idiopathic carotid inflammation seemed to exist after all.

The goal of our study was to improve the clinico-radiologic description of this unclassified entity among patients presenting with acute neck pain and abnormal carotid and pericarotidian tissues on imaging.

\section{MATERIALS AND METHODS Research Design}

We conducted a retrospective multicenter systematic chart review in centers specialized in head-and-neck and neurologic diseases. This study was approved by the institutional research ethics boards. All patients were contacted and given the opportunity to express their refusal to have their medical records used. This study follows the Strengthening the Reporting of Observational Studies in Epidemiology guidelines. ${ }^{11}$

\section{Patients}

Two physicians, a senior neuroradiologist and a neurologist, both of whom are specialized in neurovascular diseases with 30 years of experience each (J.C.S. and M.O.), screened the clinical and radiologic files of all adult patients from 10 centers who presented from January 2009 through April 2016 with acute cervical pain and with at least 1 diagnostic image (MR imaging, CT, or US) including a dedicated vessel analysis. Among patients fulfilling these criteria, they excluded cases with inadequate or incomplete vessel exploration, cases with no vascular abnormality, and those in which a clearly identified and classified vascular disease was found. The final number of patients from these centers was 47 , who all had symptomatology appropriate for the diagnosis of carotidynia and represent the study group.

\section{Review of Clinical and Biologic Charts}

Patient charts were systematically reviewed, and we collected the following clinical data: the date that pain began; the presence of major cardiovascular risk factors; medical history; medications; unilateral or bilateral cervical pain (as well as location, side, and pain scale score); a cervical swelling or a palpable abnormality over the carotid bifurcation; the presence of lymphadenopathy; the presence of associated head, neck, neurologic, or ophthalmologic symptoms; the treatment administered and its duration; the duration of pain; report of $\geq 1$ relapse during follow-up; and the total duration of follow-up. All relevant biologic data on admission and during follow-up were collected. The following relevant biologic data were recorded on admission and during follow-up for all patients in the first center (A.Rotschild Foundation's Department of Radiology): erythrocyte sedimentation rate; C-reactive protein level; complete blood count; herpes simplex virus, varicella zoster virus, Epstein-Barr virus, chlamydia, mycoplasma, influenza, human immunodeficiency virus, hepatitis B virus, hepatitis $\mathrm{C}$ virus, Lyme disease, type 1 human T-cell lymphotropic virus, and syphilis serologies, including immunoglobulin-M and immunoglobulin-G; renal function; electrolyte level; glycemia; serum cholesterol level; comprehensive hepatic workup; coagulation test; serum protein electrophoresis; thyroid hor- mone level; autoimmune markers; angiotensin-converting enzyme level; and antineutrophil cytoplasmic antibody. The minimal biologic data recorded in the other centers included erythrocyte sedimentation rate, C-reactive protein level, complete blood count, renal function, electrolytes, glycemia, serum cholesterol level, comprehensive hepatic work-up, and coagulation test.

\section{Diagnostic and Follow-Up Imaging Modalities}

US was performed with Logic E9 (GE Healthcare, Milwaukee, Wisconsin) and Xario XG (Toshiba, Tokyo, Japan) machines. Cervical and intracranial vessels were examined in B-mode and color-coded duplex with spectrum analysis.

CTA examinations were performed with a Discovery $750 \mathrm{HD}$ 64-section scanning system (GE Healthcare) in the first center, and with Discovery 750 HD 64-section or LightSpeed VCT 32section (GE Healthcare) or Somatom 16-section (Siemens, Erlangen, Germany) scanners in the other centers.

MR imaging examinations were performed with a 3T Ingenia or a $1.5 \mathrm{~T}$ Achieva imager (Philips Healthcare, Best, the Netherlands) with a 32-channel head coil covering the bifurcation in the first center and with 3T Skyra, 1.5T Avanto, 1.5T Sonata, 3T Verio (Siemens, Erlangen, Germany), 1.5T Signa Excite (GE Healthcare), or 3T Achieva (Philips Healthcare) scanners in the other centers.

Technical data are provided for the first center in On-line Tables 1 and 2.

\section{Imaging Reading Criteria}

Two neuroradiologists (A.L. and F.C. with 7 and 12 years of experience, respectively) independently analyzed the datasets in random order. The readers were blinded to the clinical and biologic data. Discrepancies were resolved by consensus.

For each diagnostic and follow-up imaging technique, the readers assessed the abnormalities as the following:

The presence of a perivascular infiltration (PVI) was defined as soft amorphous tissue replacing the fat surrounding the carotid artery, with a hazy aspect of the fat; its precise location; its dimensions (including largest axial diameter and span); its preferential side; the percentage of vessel circumference involvement; and the confidence in detecting the abnormalities $(0=$ very low level of confidence, 1 = relatively high confidence, $2=$ extremely high confidence).

The presence of vascular abnormalities, such as an intimal soft plaque, defined as a well-delineated intimal plaque, hypoechogenous without posterior acoustic shadowing in US, hypodense or hypointense compared with a normal-sized lymph node on CT or MR imaging, respectively; or a calcified plaque, defined as a hyperechogenous intimal plaque with posterior acoustic shadowing on US, isodense to the bone in CT, with no signal on MR imaging; or the presence of a lumen caliber narrowing, and its quantification by using the North American Symptomatic Carotid Endarterectomy Trial criteria. ${ }^{12}$

For US, the readers assessed the presence of a vascularization of the PVI in Doppler or power Doppler mode and the presence of cervical carotid or intracranial vessel hemodynamic changes.

For MR imaging results, the readers assessed the fat-suppressed T1- and T2-weighted imaging signal intensity of the PVI 


\begin{tabular}{|c|c|c|}
\hline Characteristics & Patients & $\%$ \\
\hline No. of patients & 47 & \\
\hline No. of carotid arteries & 49 & \\
\hline Sex ratio (women/men) & $27 / 18 ; 1.5: 1$ & \\
\hline Median age (yr) (IQR) & $48(39-56)$ & \\
\hline At least 1 vascular risk factor & 22 & $47 \%$ \\
\hline High blood pressure & 8 & $17 \%$ \\
\hline Dyslipidemia & 3 & $6 \%$ \\
\hline Mellitus diabetes & 0 & $0 \%$ \\
\hline Smoking & 19 & $40 \%$ \\
\hline History of autoimmune disease & 8 & $17 \%$ \\
\hline History of vascular event & 2 & $4 \%$ \\
\hline Recent history of viral episode & 2 & $4 \%$ \\
\hline Recent history of cervical chiropractic manipulation & 0 & $0 \%$ \\
\hline Recent history of cervical trauma & 1 & $2 \%$ \\
\hline Acute neck tenderness or cervical pain & 47 & $100 \%$ \\
\hline Affected side (left/right) & $28 / 21$ & \\
\hline Bilateral involvement & 2 & $4 \%$ \\
\hline Median pain intensity (10-point scale) (IQR) & $5(3-6)$ & \\
\hline $\begin{array}{l}\text { Cervical swelling or palpable abnormality over the } \\
\text { carotid bifurcation }\end{array}$ & 6 & $13 \%$ \\
\hline Enlarged lymph nodes at palpation & 8 & $17 \%$ \\
\hline Fever & 2 & $4 \%$ \\
\hline Flu-like symptoms & 1 & $2 \%$ \\
\hline Neurologic-associated symptoms & 8 & $17 \%$ \\
\hline No abnormal biologic test findings & 42 & $89 \%$ \\
\hline Biologic inflammatory syndrome (elevated ESR or CRP) & 3 & $6 \%$ \\
\hline Positive serum IgM & $2 / 18$ & $11 \%$ \\
\hline Treatments & 37 & $79 \%$ \\
\hline Anti-inflammatory treatment & 34 & $72 \%$ \\
\hline Steroids & 3 & $6 \%$ \\
\hline Follow-up & 47 & $100 \%$ \\
\hline Median follow-up duration (days) (IQR) & $163(88-354)$ & \\
\hline Full clinical recovery (SD) & 47 & $100 \%$ \\
\hline Mean recovery delay (days) (IQR) & $13(10-15)$ & \\
\hline Relapse & 9 & $19 \%$ \\
\hline Median delay before relapse (mo) & 6 & \\
\hline Persistence of abnormal laboratory test findings & 0 & $0 \%$ \\
\hline
\end{tabular}

Note:-ESR indicates erythrocyte sedimentation rate; CRP, C-reactive protein; IgM, immunoglobulin-M; IQR, interquartile range.

compared with a contralateral normal-sized lymph node $(1=$ less intense, 2 as intense, $3=$ more intense) and the presence of cerebral ischemic lesions with diffusion-weighted imaging and fluid-attenuated inversion recovery sequences.

For CTA and MR imaging, the readers assessed the enhancement of the PVI compared with a contralateral normal-sized lymph node $(0=$ no enhancement, $1=$ less enhancement, $2=$ same enhancement, $3=$ more enhancement) and the presence of inflammation of the pharyngeal or laryngeal mucosa.

\section{Statistical Analysis}

Results of systematic reviews were encoded on a spreadsheet and subsequently analyzed by a senior medical biostatistician (H.P.) with the R statistical package. ${ }^{13}$ Due to the descriptive design of this study, the analysis was focused on the description of patients' sociodemographic, clinical, biologic, and imaging characteristics. Interobserver agreement was assessed with nonweighted $\kappa$ statistics, by using the Landis and Koch interpretation. ${ }^{14}$ Whenever quantitative imaging variables were measured with different imaging modalities, Bland-Altman plots were used to look for an intermodality measurement bias. A linear regression was conducted to evaluate the correlation between the symptom-to- imaging delay and the following parameters: the percentage of arterial circumference involved, the presence of a lumen caliber narrowing and its severity, the PVI median largest diameter and median span, and the presence of a soft intimal plaque. A $P$ value below .05 was considered significant.

\section{RESULTS}

\section{Demographic, Clinical, and Biologic Characteristics}

Forty-seven patients presented with cervical pain, including 45 with unilateral pain and 2 with bilateral pain. The mean patient age was 48 years, and the female/male ratio was 1.5:1. Twentytwo patients $(47 \%)$ had at least 1 vascular risk factor. Eight patients (17\%) had associated neurologic symptoms: transient dizziness and vertical diplopia with extrinsic ipsilateral oculomotor cranial nerve palsy in 1 patient, a contralateral-sided dysesthesia in 4 patients, a contralateral transient motor deficit in 1 patient, and an ipsilateral peripheral facial palsy in 2 patients. Two patients had fever. Eight patients $(17 \%)$ had a clinical history of autoimmune disease: Two had ankylosing spondylarthritis, 1 had Hashimoto thyroiditis, 1 had Graves disease, 1 had systemic lupus erythematosus, 1 had Sjögren syndrome, and 2 had rheumatoid arthritis. Three patients overall (6\%) had elevated erythrocyte sediment ratios or C-reactive protein levels but normal blood counts. Two of eighteen (11\%) had elevated serum immunoglobulin-M antibodies: against herpes simplex virus for 1 patient and against type B-influenza for the other. No other patients presented with an elevation in inflammatory markers (Table 1).

\section{Diagnostic Imaging}

The median delay between the onset of symptoms and the first imaging was 5 days. All patients except 4 presented with a PVI at the level of the carotid bifurcation, most often in a posterior and lateral location (Fig 1). Two patients presented with bilateral carotid abnormalities. The median largest axial diameter of the PVI ranged from 4 to $5 \mathrm{~mm}$, and the median PVI span ranged from 15 to $28 \mathrm{~mm}$. Self-assessed confidence in investigator detection was high in all examinations performed with US or MR imaging and in $69 \%$ of the CT reviews. An intimal soft plaque was observed in 14 US reviews (58\%), 6 CT reviews (46\%), and 12 MR imaging reviews (27\%) (Fig 2). A mild lumen caliber narrowing was observed in US reviews of 9 patients (38\%), CT reviews of 4 patients (31\%), and MR imaging reviews of 12 patients (27\%). No hemodynamic change was 

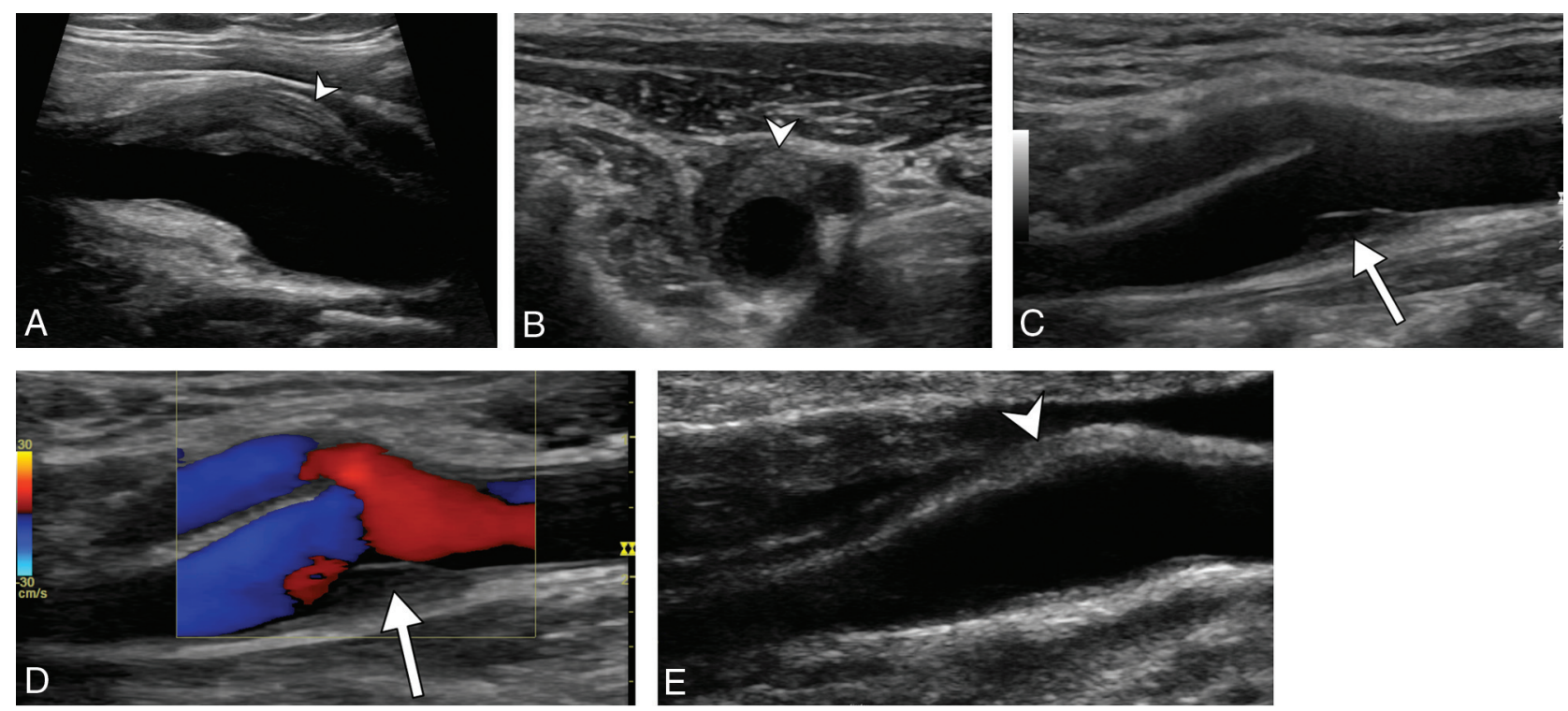

FIG 1. Diagnostic ultrasonography $(A-D)$ shows an eccentric perivascular infiltration (arrowhead) at the level of bifurcation, with a soft intimal plaque (arrow) and a mild lumen narrowing without a hemodynamic change in Doppler mode. Follow-up ultrasonography (E) shows a marked decrease in the perivascular infiltration (arrowhead) and complete disappearance of the soft intimal plaque.

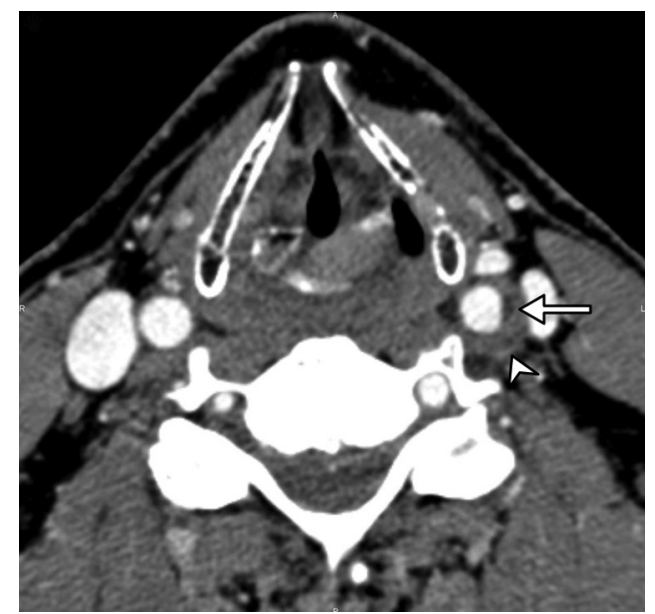

FIG 2. CTA shows a left posterolateral eccentric perivascular infiltration (arrowhead) surrounding the carotid artery, with a distinct lowdensity soft intimal plaque (arrow).

observed in color duplex Doppler. No cerebral parenchymal ischemia was seen with MR imaging.

Diagnostic imaging data are presented in Table 2.

\section{Follow-Up}

The mean follow-up duration was 3 months. All patients had a full clinical recovery, with a median delay of 13 days. Thirty-four patients received anti-inflammatory treatment, and 3 received steroids. Ten patients did not receive any treatment. Nine (19\%) patients had a clinical relapse with exactly the same clinical and imaging abnormalities. Seven of these patients had a clinical history of autoimmune disease, and they presented with a simultaneously acute exacerbation of their autoimmune disease. All laboratory test results were within normal ranges during follow-up.

Twenty-five patients (53\%) had follow-up imaging. Eleven (44\%) had US, and 23 (92\%) had MR imaging. No CTA was performed. All patients presented with a decrease or disappear- ance of the PVI; a residual PVI was noted in all patients with US and 15 patients (65\%) with MR imaging (Fig 3). Eight patients had complete disappearance of PVI on MR imaging. The median PVI diameter and span decrease ranged from 55\% to $61 \%$ and $50 \%$ to $62 \%$, respectively. Soft intimal plaque was persistent in 4 and 3 patients with US and MR imaging, respectively, and disappeared in 4 patients with US and 6 with MR imaging. Lumen caliber narrowing was persistent in 2 patients with US and 1 patient with MR imaging, respectively (Fig 4).

Follow-up imaging data are presented in Table 3.

\section{Interobserver Agreement}

Overall interobserver agreement was perfect $(\kappa=1)$ for the detection of a PVI, excellent for the PVI largest diameter or lumen caliber narrowing with US or MR imaging $(\kappa=0.9$ and 0.83 , respectively), and good for the detection of soft intimal plaque $(\kappa=0.75)$. CT showed a smaller interobserver agreement for lumen caliber narrowing and the presence of soft intimal plaque ( $\kappa=0.6$ and 0.71 , respectively).

\section{Associations with Symptom-to-Imaging Delay}

There was a significant correlation between a longer symptom-toimaging delay and a lower PVI median span $(P=.02$ and $P=.04)$ and a lower PVI median largest diameter $(P=.04)$ on sonography and a lower PVI median span $(P=.04)$ on MR imaging. There was no significant correlation between the symptom-to-imaging delay and the presence of a lumen caliber narrowing or a soft intimal plaque or the percentage of the arterial circumference involved.

\section{DISCUSSION}

We report a multicenter series of 47 patients presenting with acute neck pain and strikingly similar unclassified perivascular and vascular abnormalities seen on imaging. These findings strongly support the existence of an as yet unclassified clinico-radiologic entity 


\begin{tabular}{|c|c|c|c|c|c|c|}
\hline Characteristics & US & $\%$ & CT & $\%$ & MRI & $\%$ \\
\hline No. of patients & 23 & $49 \%$ & 13 & $28 \%$ & 43 & $91 \%$ \\
\hline No. of carotid arteries & 24 & $49 \%$ & 13 & $27 \%$ & 45 & $92 \%$ \\
\hline $\begin{array}{l}\text { Median delay between onset of symptoms and } \\
\text { diagnostic exam (days) (IQR) }\end{array}$ & \multicolumn{6}{|c|}{$5(3-7.5)$} \\
\hline High confidence in diagnosis & 24 & $100 \%$ & 9 & $69 \%$ & 45 & $100 \%$ \\
\hline Preferential side of PVI: lateral/medial & \multirow{2}{*}{\multicolumn{6}{|c|}{$\begin{array}{l}\text { 39/10: } 3.9 \\
\text { 38/11: } 3.5\end{array}$}} \\
\hline Preferential side of PVI: posterior/anterior & & & & & & \\
\hline Localization of PVI: carotid bifurcation & 23 & $96 \%$ & 12 & $92 \%$ & 41 & $91 \%$ \\
\hline Circumferential & 0 & $0 \%$ & 0 & $0 \%$ & 2 & $4 \%$ \\
\hline $\begin{array}{l}\text { Percentage of carotid circumference involved } \\
\text { (\%) (IQR) }\end{array}$ & $40(30-40)$ & & $30(20-40)$ & & $40(30-57.5)$ & \\
\hline PVI & 24 & $100 \%$ & 13 & $100 \%$ & 45 & $100 \%$ \\
\hline Pericarotid fat stranding & 24 & $100 \%$ & 13 & $100 \%$ & 45 & $100 \%$ \\
\hline Lumen caliber narrowing & 9 & $38 \%$ & 4 & $31 \%$ & 12 & $27 \%$ \\
\hline Mean percentage of carotid stenosis (\%) (IQR) & $30(20-30)$ & & $22.5(18-26)$ & & $20(14-30)$ & \\
\hline Mean PVI span (mm) (IQR) & $15(10-22)$ & & $15(10-26)$ & & $28(17-34)$ & \\
\hline Median PVI largest diameter (mm) & $4(2.4-6)$ & & $4(3.6-4.8)$ & & $5(4-7)$ & \\
\hline Soft intimal plaque & 14 & $58 \%$ & 6 & $46 \%$ & 12 & $27 \%$ \\
\hline Enlarged lymph nodes & 8 & $33 \%$ & 0 & $0 \%$ & 8 & $18 \%$ \\
\hline PVI enhancement & NA & & 11 & $85 \%$ & 45 & $100 \%$ \\
\hline Laryngeal or pharyngeal inflammation & 0 & $0 \%$ & 0 & $0 \%$ & 1 & $2 \%$ \\
\hline
\end{tabular}

Note:-IQR indicates interquartile range; NA, not applicable.
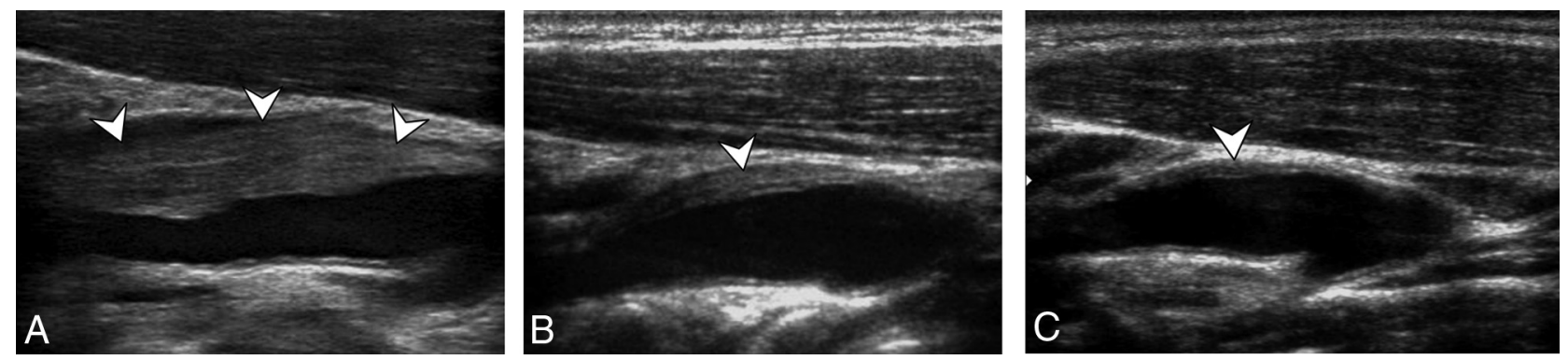

FIG 3. Initial diagnostic ultrasonography $(A)$ and follow-up ultrasonography at 14 days $(B)$ and 6 months $(C)$ show a perivascular infiltration (arrowheads) at the level of the internal carotid artery just at the level of bifurcation, with a quick decrease at 14 days and the persistence of a thin abnormality at 6 months.

classically diagnosed as "carotidynia" in the literature dated before 2004 .

\section{Clinical and Imaging Characteristics}

This entity was relatively rare in our study, with an estimated prevalence of $2.8 \%$ (18/654) among patients with acute neck pain in the first center. However, we can presume that this particular cluster of symptoms is underestimated because of the relatively mild clinical symptoms and the quick relief of pain and decrease of imaging abnormalities within 13 days. This entity has been previously reported on in a few small series as carotidynia, but we present the largest series published so far with 3 distinct imaging modalities and follow-up data.

Clinically, all our patients presented with acute pain directly around the level of the carotid bifurcation. Eight patients presented with previously undescribed, transient neurologic symptoms, which lacked explanation despite a brain parenchyma MR imaging. After imaging, the presence of a unilateral eccentric PVI at the level of bifurcation was the most striking feature described. ${ }^{6,7,10}$ Two patients presented with bilateral carotid involvement, ${ }^{9}$ though most were unilateral. ${ }^{6,7}$ PVI imaging characteristics were similar to those found in the literature, with a median diameter of $5 \mathrm{~mm}$ and a median span of $20 \mathrm{~mm} .^{7,15}$
Some patients had a mild associated narrowing of the lumen, ${ }^{6,16}$ though most patients had no luminal change ${ }^{7,10}$ or any hemodynamic abnormality.

We report the presence of a self-limited, intimal soft plaque in half of our patients, which has been described in previous reports. ${ }^{6,17}$ These intimal changes might be induced by the healing phase of the carotid inflammatory process.

Nonsteroidal anti-inflammatory agents and high doses of aspirin were the most frequent treatment in our population and in the literature, with a complete median delay of pain relief in 13 days. ${ }^{5-7}$ Some authors reported full recovery with clopidogrel ${ }^{8}$ or without any treatment at all. ${ }^{7}$ All patients had complete clinical resolution during follow-up. Nine patients presented with $\geq 1$ relapse with intervals ranging from 1 to 6 months, similar to what is found in the literature. ${ }^{18}$

Although early case reports described the complete disappearance of clinical and imaging abnormalities, ${ }^{7}$ more recent case reports showed a persistence of imaging abnormalities, similar to our findings. ${ }^{6,17,19}$ It could be explained by the development of early fibrosis associated with low-grade chronic active inflammation, as described in the only histologic study found during the literature review. ${ }^{20}$ 

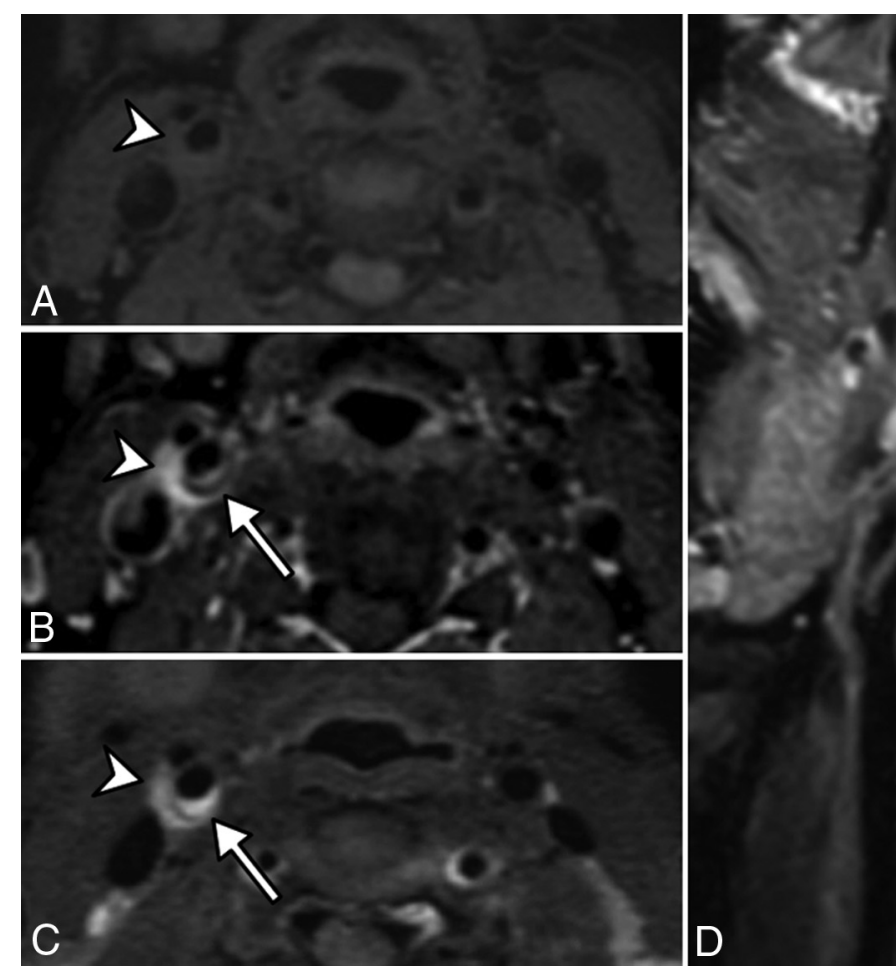

FIG 4. Pre $(A)$ and post $(B)$ contrast fat-suppressed 3D T and 3D T2-weighted (C) MR imaging in an axial plane shows $T 1$ hypointense and T2 hyperintense perivascular infiltration (arrowhead) at the level of the carotid artery bifurcation, enhanced after gadolinium injection. A distinct soft intimal plaque (arrow) is visible at the posterior part of the carotid artery. A sagittal curvilinear reconstruction of the right internal carotid artery on the postcontrast T-weighted imaging $(D)$ shows the PVI (arrowhead) centered at the level of the right carotid artery bifurcation and extended to both the distal common carotid artery and proximal internal carotid artery. Note that there is no vascular or perivascular abnormality involving other parts of the common or the internal carotid arteries.

Table 3: Follow-up imaging data

\begin{tabular}{|c|c|c|c|c|}
\hline Characteristics & US & $\%$ & MRI & $\%$ \\
\hline No. of patients & 11 & $44 \%$ & 23 & $51 \%$ \\
\hline $\begin{array}{l}\text { Median delay between onset of symptoms and } \\
\text { follow-up exam (days) (IQR) }\end{array}$ & \multicolumn{4}{|c|}{89 (71.5-171) } \\
\hline PVI & 11 & $100 \%$ & 15 & $65 \%$ \\
\hline Complete PVI disappearance & 0 & $0 \%$ & 8 & $35 \%$ \\
\hline PVI decrease & 11 & $100 \%$ & 15 & $65 \%$ \\
\hline PVI stability & 0 & $0 \%$ & 0 & $0 \%$ \\
\hline $\mathrm{PVI}$ increase & 0 & $0 \%$ & 0 & $0 \%$ \\
\hline Lumen caliber narrowing & 2 & $18 \%$ & 1 & $4 \%$ \\
\hline Median PVI largest diameter (mm) (IQR) & \multicolumn{2}{|c|}{$1.5(0.9-2.4)$} & \multicolumn{2}{|c|}{$2.9(2.0-3.5)$} \\
\hline Median decrease of the PVI largest diameter & \multicolumn{2}{|c|}{$61 \%$} & \multirow{2}{*}{\multicolumn{2}{|c|}{$\begin{array}{c}55 \% \\
13(9.5-16)\end{array}$}} \\
\hline Median PVI span (mm) (IQR) & \multicolumn{2}{|c|}{$9(5-10)$} & & \\
\hline Median decrease of the PVI span & & & \\
\hline Persistence of a soft intimal plaque & 4 & $50 \%$ & 3 & $33 \%$ \\
\hline Disappearance of a soft intimal plaque & 4 & $50 \%$ & 6 & $67 \%$ \\
\hline PVI enhancement & NA & & 12 & $52 \%$ \\
\hline
\end{tabular}

\section{Possible Pathophysiologic Mechanisms}

Most case reports and small studies hypothesized that observed vascular and perivascular changes were consistent with inflammation. ${ }^{9,19}$ Clinical findings support this hypothesis, with ipsilateral lymph node enlargement and/or contiguous pharyngolaryngeal inflammation, ${ }^{21}$ as well as biologic findings, with a mild increase of the erythrocyte sedimentation rate or C-reactive protein. ${ }^{8,9,22,23}$ In our series, only 3 patients had a mild increase of the erythrocyte sedimentation rate or C-reactive protein levels. Eight patients had an autoimmune disease such as rheumatoid arthritis, systemic lupus erythematosus, ankylosing spondylarthritis,

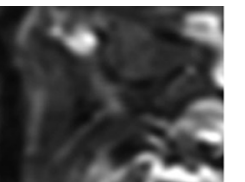

Graves disease, Sjögren syndrome, or Hashimoto thyroiditis. Most interesting, 7 over these 8 patients presented with several simultaneous clinical relapses of the perivascular inflammation of the carotid artery and of their autoimmune disease, suggesting a link between the diseases. One study reported a case of fluoxetine-induced carotidynia, presumably explained by the inflammatory modulation induced by the antidepressants. ${ }^{24}$ Some studies reported an increased activity corresponding to the region of soft-tissue thickening within the carotid sheath with FDGPET-CT, ${ }^{8,9,25}$ though these findings were not specific for inflammation. Other hypotheses were discussed, such as vasculitis. ${ }^{26}$ However, PET-CT with abnormally increased activity in neither the remainder of the body nor the vessels was described. ${ }^{8,9}$ In our center, none of the patients with biopsy-proved giant cell arteritis presenting with acute neck pain or tenderness had abnormalities of the carotid bulb on imaging, and no relationship between the 2 entities was reported in literature. Spontaneous resolution excludes neoplastic processes. Finally, 1 study reported histologically proved findings of chronic inflammation in 1 patient with pathologic changes consisting of vascular and fibroblast proliferation and predominantly lymphocytic low-grade chronic active inflammation. ${ }^{20}$ Therefore, given that the pathogenesis remains unknown, this entity may be an inflammatory process of unknown origin or part of an autoimmune process.

\section{Proposition for a New Entity}

In 2004, carotidynia was removed from the International Classification of Headache Disorders. ${ }^{3}$ Causes of acute neck pain are numerous, ${ }^{27}$ and we agree that the term "carotidynia" is confusing and should not be used anymore. ${ }^{4}$ However, our study strongly suggests the existence of a clinical entity that was formerly poorly described (often under the "carotidynia" label) with very consistent clinical and imaging characteristics. In our study, the attempt to label this entity was shown to be dysfunctional and confusing among the different centers with at least 4 distinct labels used to describe the same entity ("carotidynia," "carotidodynia," "carotidobulbia," or "carotiditis"). In the past, some authors proposed terms such as "idiopathic carotiditis" ${ }^{19}$ or "carotid periarteritis," these terms were not accurate because vascular and perivascular abnormalities occur simultaneously. 
Consequently, we strongly support the need for a new label, and we suggest an acronym most apt for this entity: TransIent Perivascular Inflammation of the Carotid artery (TIPIC) syndrome. This acronym was chosen by consensus of the neurologists, radiologists, internists, and vascular physicians working at the different centers that collaborated on this project.

We believe that this entity should be added to the International Classification of Headache Disorders-III. ${ }^{28}$ We propose 4 major criteria as follows:

1) Presence of acute pain overlying the carotid artery, which may or may not radiate to the head

2) Eccentric PVI on imaging

3) Exclusion of another vascular or nonvascular diagnosis with imaging

4) Improvement within 14 days either spontaneously or with anti-inflammatory treatment.

Additionally, a minor criterion could be the presence of a selflimited intimal soft plaque.

\section{Limitations of the Study}

Our study has some limitations. First, it was a retrospective, descriptive study with a small number of patients, thus limiting its scope mostly in the descriptive and exploratory fields. Second, a non-negligible number of patients were probably not selected during the screening process because of a lack of diagnostic imaging or a delayed order for diagnostic imaging. Third, our median follow-up duration was short, and we could not say whether follow-up imaging would eventually show the complete disappearance of the PVI, relapses, or development of atherosclerosis. Fourth, because of the benign course of the disease, adding details from pathology is difficult and not feasible; thus, better comprehension of the physiopathology has been stalled for the moment.

\section{Changes in Current Practice}

We believe that clinicians should think about the TIPIC syndrome in the differential diagnosis of neck pain. They could set a precise diagnosis with adequate imaging modalities, to exclude other entities in the differential diagnosis and propose adequate treatment. Recognition of this syndrome would be cost-effective and avoid unnecessary, additional diagnostic examinations. US appears to be a suitable examination for screening because it can detect PVI with high accuracy, similar to other imaging methods, but without any exposure to radiation, high magnetic fields, or the administration of intravenous contrast agents. It is affordable and easily accessible, and it has excellent interreader concordance. Imaging diagnosis should be performed without delay because these abnormalities decrease very quickly. Follow-up could be made with US to assess PVI decrease and control lumen narrowing or soft intimal plaque. ${ }^{5}$

Our study raised concerns about 2 major issues. First, the neurologic risk may not be as low as previously reported in the literature because 8 of our patients had neurologic events. We do not have a convincing explanation regarding the possible relationship between this entity and the neurologic events encountered. The lumen caliber narrowing was mild, and there were no local or distal hemodynamic changes with cervical or transcranial US. Brain MR imaging performed at the time of the neurologic events did not show any acute brain abnormality with diffusion or FLAIR-weighted imaging. Therefore, the hypothesis of a brain ischemia linked to the carotid artery narrowing, either due to distal embolism or low-flow, is unlikely. An inflammatory hypothesis involving both the carotid artery and intracranial arteries at the same time might be considered, but our imaging protocol was not designed to detect intracranial vessel wall inflammation.

Second, the persistence of vascular wall abnormalities, including an intimal soft plaque with imaging, suggest the hypothesis of a secondary development of atherosclerosis in these patients, therefore justifying a longer follow-up.

Recognition of this entity favors further, large prospective studies to answer these major questions and to elucidate the prevalence, pathophysiologic mechanisms, risk factors, etiologic processes, and potential therapies for this condition.

\section{CONCLUSIONS}

We describe more precisely a currently unclassified clinico-radiologic entity in patients presenting with acute cervical pain and strikingly similar perivascular and vascular abnormalities during diagnostic imaging. We suggest a suitable name to describe this entity: TransIent Perivascular Inflammation of the Carotid artery (TIPIC) syndrome.

\section{ACKNOWLEDGMENTS}

Laura McMaster provided professional English-language medical editing of this article.

Disclosures: Julien Savatovsky—UNRELATED: Consultancy: Servier International Research Institute; Grants/Grants Pending: AIDS National Research Agency*; Payment for Lectures Including Service on Speakers Bureaus: Bayer, Medtronic, Philips Healthcare; Travel/Accommodations/Meeting Expenses Unrelated to Activities Listed: Bayer, Philips Healthcare, GE Healthcare. *Money paid to the institution.

\section{REFERENCES}

1. Fay T. Atypical neuralgia. Arch Neurol Psychiatry 1927;18:309-15

2. Classification and diagnostic criteria for headache disorders, cranial neuralgias and facial pain: Headache Classification Committee of the International Headache Society. Cephalalgia 1988;8(suppl 7): 1-96 CrossRef Medline

3. Headache Classification Subcommittee of the International Headache Society: The International Classification of Headache Disorders-2nd edition. Cephalalgia 2004;24(suppl 1):9-160 CrossRef Medline

4. Biousse V, Bousser MG. The myth of carotidynia. Neurology 1994; 44:993-95 CrossRef Medline

5. Behar T, Menjot N, Laroche J-P, et al. Comparative evolution of carotidynia on ultrasound and magnetic resonance imaging. J Mal Vasc 2015;40:395-98 CrossRef Medline

6. Arning C. Ultrasonography of carotidynia. AJNR Am J Neuroradiol 2005;26:201-02 Medline

7. Burton BS, Syms MJ, Petermann GW, et al. MR imaging of patients with carotidynia. AJNR Am J Neuroradiol 2000;21:766-69 Medline

8. Amaravadi RR, Behr SC, Kousoubris PD, et al. [18F] fluorodeoxyglucose positron-emission tomography-CT imaging of carotidynia. AJNR Am J Neuroradiol 2008;29:1197-99 CrossRef Medline

9. Hafner F, Hackl G, Haas E, et al. Idiopathic carotidynia. Vasa 2014; 43:287-92 CrossRef Medline

10. Kosaka N, Sagoh T, Uematsu H, et al. Imaging by multiple modalities of patients with a carotidynia syndrome. Eur Radiol 2007;17: 2430-33 CrossRef Medline

11. Vandenbroucke JP, von Elm E, Altman DG, et al; STROBE Initiative. Strengthening the Reporting of Observational Studies in Epidemi-

AJNR Am J Neuroradiol 38:1391-98 Jul 2017 www.ajnr.org

1397 
ology (STROBE): explanation and elaboration. PLoS Med 2007;4: e297 CrossRef Medline

12. Clinical alert: benefit of carotid endarterectomy for patients with high-grade stenosis of the internal carotid artery-National Institute of Neurological Disorders and Stroke Stroke and Trauma Division. North American Symptomatic Carotid Endarterectomy Trial (NASCET) investigators. Stroke 1991;22:816-17 CrossRef Medline

13. R Foundation for Statistical Computing, Vienna, Austria. http:// www.R-project.org/. 2016

14. Landis JR, Koch GG. An application of hierarchical kappa-type statistics in the assessment of majority agreement among multiple observers. Biometrics 1977;33:363-74 CrossRef Medline

15. Kuhn J, Harzheim A, Horz R, et al. MRI and ultrasonographic imaging of a patient with carotidynia. Cephalalgia 2006;26:483-85 CrossRef Medline

16. Wu Z, Yang H. Color Doppler imaging in the diagnosis and follow-up of carotid cavernous sinus fistulas. Yan Ke Xue Bao 1993;9: 153-57 Medline

17. Woo JK, Jhamb A, Heran MK, et al. Resolution of existing intimal plaque in a patient with carotidynia. AJNR Am J Neuroradiol 2008; 29:732-33 CrossRef Medline

18. Young JY, Hijaz TA, Karagianis AG. CT findings in a patient with bilateral metachronous carotidynia. Clin Imaging 2015;39:305-07 CrossRef Medline

19. Tardy J, Pariente J, Nasr N, et al. Carotidynia: a new case for an old controversy. Eur J Neurol 2007;14:704-05 CrossRef Medline
20. Upton PD, Smith JG, Charnock DR. Histologic confirmation of carotidynia. Otolaryngol Head Neck Surg 2003;129:443-44 CrossRef Medline

21. Comacchio F, Bottin R, Brescia G, et al. Carotidynia: new aspects of a controversial entity. Acta Otorhinolaryngol Ital 2012;32:266-69 Medline

22. da Rocha AJ, Tokura EH, Romualdo AP, et al. Imaging contribution for the diagnosis of carotidynia. J Headache Pain 2009;10:125-27 CrossRef Medline

23. Taniguchi $\mathrm{Y}$, Horino $\mathrm{T}$, Hashimoto $\mathrm{K}$. Is carotidynia syndrome a subset of vasculitis? J Rheumatol 2008;35:1901-02 Medline

24. Jabre MG, Shahidi GA, Bejjani BP. Probable fluoxetine-induced carotidynia. Lancet 2009;374:1061-62 CrossRef Medline

25. Berzaczy D, Domenig CM, Beitzke D, et al. Imaging of a case of benign carotidynia with ultrasound, MRI and PET-CT. Wien Klin Wochenschr 2013;125:719-20 CrossRef Medline

26. Taniguchi $\mathrm{Y}$, Horino $\mathrm{T}$, Terada $\mathrm{Y}$, et al. The activity of carotidynia syndrome is correlated with the soluble intracellular adhesion molecule-1 (sICAM-1) level. South Med J 2010;103:277-78 CrossRef Medline

27. Chambers BR, Donnan GA, Riddell RJ, et al. Carotidynia: aetiology, diagnosis and treatment. Clin Exp Neurol 1981;17:113-23 Medline

28. Headache Classification Committee of the International Headache Society (IHS): The International Classification of Headache Disorders-3rd edition (beta version). Cephalalgia 2013;33:629-808 CrossRef Medline 